\title{
Applying local Green's functions to study the influence of the crustal structure on hydrological loading displacements
}

\author{
Robert Dill and Volker Klemann \\ GFZ, Helmholtz Centre Potsdam, 1.3 Earth System Modelling, Potsdam, Germany (dill@gfz-potsdam.de)
}

The influence of the elastic Earth properties on seasonal or shorter periodic surface mass loads due to atmospheric surface pressure and terrestrial water storage variations is usually modeled by applying a local isostatic model like a homogeneous half-space model, or by a one dimensional spherical Earth model like PREM from which a unique set of elastic load Love numbers, or alternatively, elastic Green's functions are derived. The drawbacks of these strategies are that, in the first case, the response according to the local Earth structure is valid only if load and observer almost coincide, or that, in the second case, only the response of an average Earth structure is considered. However, for surface loads with horizontal scales less than $2500 \mathrm{~km}^{2}$, as for instance, for strong localized hydrological signals associated with heavy precipitation events and river floods, the Earth elastic response becomes very sensitive to inhomogeneities in the Earth crustal structure.

We derive a set of local Green's functions defined for every global $1^{\circ} \times 1^{\circ}$ gridcell for the 3-layer crustal structure TEA12. Local Green's functions show standard deviations of $\pm 12 \%$ in the vertical and $\pm 21 \%$ in the horizontal directions for distances in the range from $0.1^{\circ}$ to $0.5^{\circ}$. The application of local Green's functions introduces a variability of $0.5-1.0 \mathrm{~mm}$ into the hydrological loading displacements, both in vertical and in horizontal directions. Maximum changes due to the local crustal structures are from $-25 \%$ to $+26 \%$ in the vertical and $-91 \%$ to $+55 \%$ in the horizontal displacements. In addition, the horizontal displacement changes its direction significantly, even to the opposite. The modeling of a site-dependent crustal response to surface loads provides an alternative way to probe the density and elastic structure of the Earth's crust and mantle by means of observed surface deformations caused by mass re-distributions. In addition, realistic loading models allow the monitoring of mass variations of the hydrosphere and cryosphere in the spatial range between satellite resolution and in-situ observations by the analysis of geodetically measured surface displacements. 